\title{
Patterns of care study for brachytherapy: results of the questionnaire for the years 2002 and 2007 in The Netherlands
}

\author{
Jack L.M. Venselaar, PhD', Ben J. Slotman, MD, Prof., PhD², Ferran Guedea, MD, Prof., PhD³, Montse Ventura², \\ Bradley Londres ${ }^{3}$, Guy Francois ${ }^{4}$ \\ IInstituut Verbeeten, Tilburg, The Netherlands, 2VU University Medical Center, Amsterdam, The Netherlands, ${ }^{3}$ nnstitut Catala d'Oncologia, \\ Barcelona, Spain, ${ }^{4}$ ESTRO Office, Brussels, Belgium
}

Research conducted at:

Instituut Verbeeten, Dept Medical Physics, Tilburg, The Netherlands

\begin{abstract}
Purpose: The goal of the ESTRO Patterns of Care study for Brachytherapy in Europe (PCBE) 2002 was to develop an aid to analyse brachytherapy practices. A $2^{\text {nd }}$ version of the PCB questionnaire was created for 2007. Data over 2007 were collected at the radiotherapy institutions in The Netherlands and compared with those from 2002. The aim of this study is to describe national brachytherapy practices, to demonstrate trends, and to provide data for rational health care planning.

Material and methods: Data were collected using a web-based questionnaire. For each centre, a local coordinator, responsible for coordinating the questionnaires and support of the further analysis was assigned. Data from the national cancer incidence registry was used for comparison with the data from the 21 Dutch departments.

Results: There was a decrease in low-dose rate equipment in parallel to an increase in both pulsed-dose rate and high-dose rate equipment. The use of 3D CT and MR based imaging techniques showed a slow rise. The most common clinical procedures were for prostate, gynaecological, and oesophageal tumours. A large increase (146\%) in permanent implant prostate applications using ${ }^{125}$ I seeds was observed. The numbers of oesophageal and gynaecological treatments remained stable. There is concern on the low numbers of cases treated in some institutions for a few complex treatment sites. For head and neck, anal canal, paediatrics, bladder and eye interventions it ranged from 3-20 patients per year per institution.

Conclusions: The increase in number of patient treated with brachytherapy is in accordance with the increases in cancer incidence. The percentage of all radiotherapy patients treated with brachytherapy (approximately 5\%) remained stable. The survey identified certain trends in resources and techniques, as well as areas of expected improvement and possible gain in clinical outcome. Data reported from this survey can be used for further planning of resources, facilities and concentration of a low-volume specialised and complex treatments.
\end{abstract}

Key words: patterns of care study, brachytherapy, The Netherlands.

\section{Purpose}

During the 2003 GEC-ESTRO (Groupe Européen de Curiethérapie-European Society for Therapeutic Radiation Oncology) meeting held in Lübeck, Germany, the steering committee decided to launch the Patterns of Care Study for Brachytherapy in Europe (PCBE). The primary aim of this project was to perform a survey of brachytherapy (BT) facilities in the European area. This data was considered crucial for the completion of three other ESTRO projects: ESQUIRE (Education, Science and Quality Assur- ance in Radiotherapy in Europe), QUARTS (Quantification of Radiotherapy Infrastructure and Staffing), and EMIR (European Network for Medical Radioisotope and Beam Research). The QUARTS project aimed at providing health care authorities with objective criteria for infrastructure and staffing needs for radiotherapy (RT). A quantitative analysis of demands on staff and infrastructure per tumour site/type and of a population-based hierarchy of treatment facilities, adapted to local epidemiology and staffing costs, was performed to assist in prioritising financial resources [1,2]. 
The objective of the PCBE was to create a detailed information system specifically on the BT practices and resources throughout Europe and to monitor changes over time to identify trends and developments in the field of BT. The data thus gathered might further support or develop European policies for improvement of BT care planning in the EU based on rational grounds. An analysis of the acquired national data may show changes over time in available therapeutic or diagnostic equipment, staffing levels, numbers of patients treated, and compliance to (inter)national recommendations and protocols. Examples include changes in technical resources, such as the introduction and use of CT, MR and PET imaging and the phasing out of low-dose rate afterloading equipment in favour of high-dose rate afterloaders.

A first report of the 2002 data of the PCBE project was published in 2007 [3]. In that study, 100\% of institutions in The Netherlands provided their data [4]. Similar overviews from other EU countries on BT facilities have been published [5-8]. The $2^{\text {nd }}$ version of the survey for 2007 repeated most of the 2002 questions, but had a special focus on treatment of lung, breast, gynaecological, and prostate cancer patients. In addition, the general project was extended to include countries in Latin America. For this reason, the name of the project was changed to the Patterns of Care study for Brachytherapy (PCB). The value of the data collected is more valuable now that we have results from 2002 and 2007, thus allowing for the identification of changes over this 5-year interval, and especially considering that the number of participating countries has grown. A total of 62 countries agreed to be involved in the data collection. General results will be presented elsewhere [9].

In the present overview, we describe the results of the surveys for the years 2002 and 2007 in The Netherlands. The distribution of BT facilities and the available resources are analysed and trends and developments therein are identified.

\section{Material and methods}

Both the 2002 and 2007 surveys focussed on the following classes of items: (i) general issues: number of institutions and patients, treatments performed, and staff performing BT; (ii) resources available for BT; (iii) participation in research and educational programmes; (iv) number of BT patients, with a breakdown by technique and body site; (v) specific questions on BT for lung and breast tumours, and detailed questions on gynaecological and prostate treatments. Essentially the questions in the new 2007 survey were the same as in 2002, with a number of minor improvements [9]. Some new questions were added - e.g. on the use of MR and PET imaging for BT - in response to technological developments in these areas. Importantly, a series of site-specific questions on the procedures for several tumour sites were added, as follows: questions related to treatment intent for endoluminal lung tumours, technical procedures used for breast tumours. In addition, for gynaecological tumours, questions were included about treatment intent, type of planning procedure, and whether or not the GEC-ESTRO recommendations were followed.
For prostate, questions were included on the availability of other techniques, the use of LDR vs. PDR and HDR, the use of loose vs. stranded seeds for permanent implant techniques, and on dose prescription.

The organization of the survey was very similar to 2002 [3], with a local coordinator in each of the participating countries and a web-based document system, so that individual institutions could input the data directly. The online questionnaire was hosted at the ESTRO web site, while the study was coordinated by staff located at the Institut Catala d'Oncologia in Barcelona, Spain. The questionnaire is available as an appendix to the web version of the paper of the general results in Europe [9].

The data collected was either from the reporting period of January 1 through December 31 of the given year, or the status of the department at the end of the year. For the 2002 data, 13 of 22 institutions in The Netherlands input their data via the web while the remaining centres sent their material by fax or email to the national coordinator. In the 2007 study, access to the new website for data collection was much improved and all responses were entered into the web database directly by the institutions. The Dutch national radiation oncology organization (NVRO) agreed to participate in the 2007 study and will likely do so again if the study is continued in the future years.

\section{Geographical information}

The Netherlands has approximately 16.5 million inhabitants. RT is performed at all university hospitals (8), some of the larger hospitals (9), and at several independent RT institutions (5). In 2002, there were a total of 22 RT departments in the country, although that number was later reduced to 21 when one of the independent institutions merged with a university hospital. The facilities are reasonably well-spread geographically throughout the country. A 100\% response rate was achieved. The Dutch Comprehensive Cancer Centres publish annually the incidence rate of invasive tumours in a cancer registry with updates through the year 2007, last accessed in May 2010 [10]. Cancer incidence rates for The Netherlands mentioned in this paper are taken from this registry.

\section{Definitions}

For patients and treatments, the following definitions were used. RT Patients: A patient who has a treatment with external beam radiotherapy (EBRT) and/or BT. A person is included as one RT patient, even if the treatment is a combination of these two modalities. If a new site is treated within the same (calendar) year, this person is included as two RT patients. Brachytherapy patients: A person is included as one BT patient, even if the treatment is combined with EBRT. If a new site is treated with BT within the same year, this person is included as two BT patients. Brachytherapy application: If needles and/or applicators are inserted and this implant is used for several fractions it will be included as one application. If the needles and/or the applicators are removed and inserted several days or weeks later, this is included as two BT applications. 


\section{Results}

\section{Patients, applications, and staff}

Figure 1 shows the number of RT patients for all institutions in 2002 and 2007. The total number of RT patients increased from 41528 in 2002 to 47594 in 2007. The average number of patients per institution is 2266 , but the spread is considerable (varying from 700-4600), as can be seen in the histogram. The average increase of the number of RT patients over the 5 year interval was close to $15 \%$, but this was highly variable over the institutions. The comprehensive cancer centre registry data base listed a total of 73675 invasive cancer cases in 2002 and 86162 in 2007, an increase of $17 \%$ [10].

Figure 2 shows for all institutions the overall numbers of BT patients and applications separated for 2002 and 2007. A total of 2460 BT patients and 3392 BT applications were reported for 2007. This is compared with 1987 BT patients and $2460 \mathrm{BT}$ applications in 2002. The average number of patients per institution was 123 , but the standard deviation was considerable (89.8 SD). In 2007, the number of BT patients per institution ranged from 29 to 329 , with a median of 112 patients. The ratio of applications per patient increased from 1.24 to 1.38 over the five year period, indicating on average a higher fractionation of treatments.

Both the 2002 and the 2007 survey included questions on the number of consultant radiation oncologists (trainees excluded) at each institution, and the number of these that regularly perform $\mathrm{BT}$ procedures, expressed as full time equivalents (FTE). These data show that the number of radiation oncologists in The Netherlands increased from 152 in 2002 to 196 in 2007, an increase of about 29\%, much higher than the increase of the number of RT patients treated in those years (Fig. 1). The number of radiation oncologists performing BT at the institutions was basically stable, with 84 in 2002 and 87 in 2007.

\section{Resources}

In 2002, a total of 36 afterloaders were available at Dutch RT centres, an average of 2 afterloaders per institution. In 2007, the total number of installed afterloaders in the country fell to 29. Most of the low dose-rate (LDR) afterloaders were decommissioned: out of 7 Micro-LDR systems only one remained, while the number of LDR $\left({ }^{137} \mathrm{Cs}\right.$ pellet based) afterloaders dropped from 9 to 2 systems. The use of HDR and PDR afterloaders increased from 13 to 15 and from 7 to 11 machines, respectively. The manual wire technique was employed in 5 institutions in 2002 and in 4 in 2007. Permanent prostate implants using (only) ${ }^{125}$ I seeds was performed in 10 institutions in 2002, and in 13 in 2007. Six institutions used eye applicators in 2007. Endovascular BT was discontinued and no longer employed in 2007.

The shift from LDR to HDR treatment was paralleled by changes in the availability of other resources: the number of specialised operating rooms available in the BT units increased from 6 to 11 of the 20 BT institutions, while the total number of dedicated shielded patient rooms decreased from 44 to 36 . The routine use of 3D imaging for

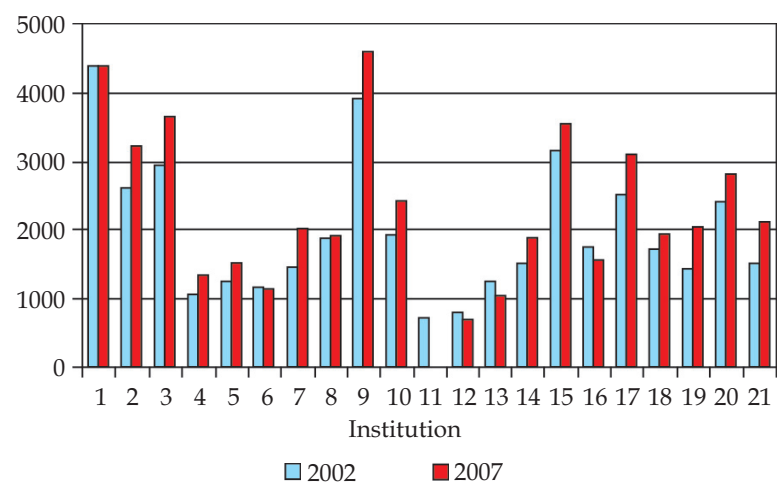

Fig. 1. The number of new radiotherapy patients treated in the institutions in The Netherlands in 2002 and 2007

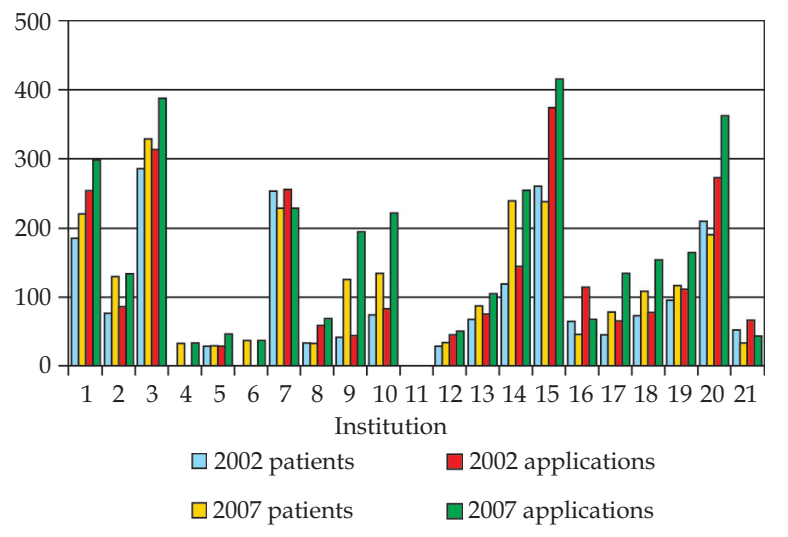

Fig. 2. The number of new patients treated with brachytherapy in The Netherlands in 2002 and 2007 (left two bars for each institution number), and the number of applications in 2002 and 2007 respectively (right two bars)

dosimetry using CT increased from 6 to 10 institutions. Use of MRI scanning increased from 3 to 4 . The use of a planar film technique, 3D ultrasound, and PET scanning was asked only in the 2007 survey, showing that 13,14, and 1 institutions used these techniques, respectively. The number of institutions applying in-vivo dosimetry during the procedures rose from 2 to 4 . The number of treatment planning systems (TPSs) for use with BT (not workstations but rather the TPSs actually used for clinical BT) increased from 26 to 40 between 2002 and 2007.

\section{Research and education}

Eight of the 20 Dutch institutions provided information on research activities, reporting participation in a total of 4 prospective clinical trials and 6 study protocols. Several centres reported participation in the PORTEC-2 (4 centres) and PORTEC-3 (1 centre) trials [11,12], and three reported involvement in the imaging-based cervix programme of the GEC-ESTRO Embrace group [13,14].

Only 4/20 institutions provided - free text - information about educational programmes offered, and such programmes ranged from practical training of residents, participation of trainees in ESTRO teaching courses, to the organization of a PDR workshop. 


\section{Site specific patient numbers}

Table 1 shows patient numbers per tumour category for the years 2002 and 2007, along with the number of institutions where the techniques are employed and the average number of patients treated at each institution. Several indications are widely spread over the institutions (gynaecological, prostate, oesophagus), while most others are performed only at a limited number of institutions, with few cases (paediatrics, bladder, anal-rectum). Several indications (eye, breast, head \& neck) are concentrated in a small number of centres.

The most striking differences between 2002 and 2007 are the increase in prostate implants, and the decrease in lung/bronchus (from 30 to 3), and, especially, endovascular treatments: the number of endovascular procedures dropped from 148 in 2002 (recorded as "others" in the 2002 column) to zero in 2007. The number of skin treatments decreased considerably, although it should be noted that the 2002 data did not distinguish between treatments for skin cancer and benign keloids: 44 patients with skin treatment were recorded in 2002 vs. 14 skin cancers and 26 keloid treatments in 2007.

The last column of the table shows the 2007 cancer incidence in The Netherlands [10]. The $3^{\text {rd }}$ column lists the percentage of invasive cancer incidence treated with BT.

\section{Detailed site-specific questions}

In 2007, only 79 patients from 4 different centres underwent breast BT, with 67 cases $(85 \%)$ treated at a single centre. The 2002 data showed a similar pattern, with 72 breast patients treated at 5 institutions, 48 of whom were treated at a single centre. Nearly all treatments (78 of 79 cases) were boost BT with one exception (a chest wall recurrence). A multi-catheter technique was used in all cases.

In 2007, 596 patients were treated for gynaecological disease, almost the same as in 2002 (586). A breakdown by tumour site and treatment intent is shown in Table 2. Endometrial BT was postoperative, whereas cervix and vagina techniques are mainly definitive. In 2007, all preoperative planning in The Netherlands was performed with conventional 2D orthogonal $\mathrm{X}$-ray reconstruction techniques. Of all postoperative and definitive case planning $43 \%$ were performed with 3D CT (33\% of cases) or MR imaging $(10 \%)$. The rest was still based on 2D X-ray based planning.

In total, 1030 prostate patients were treated in 2007 using ${ }^{125}$ I seeds (vs. 419 in 2002), while 20 cases were treated with PDR and 22 with HDR (exactly the same as in 2002). The number of institutions performing prostate implants grew from 9 in 2002 to 13 in 2007, with 7 of the original 9 reporting large increases in patient numbers, and the other two reporting little change (one institution reported a decrease from 6 to 0 cases). Four centres started completely new prostate programmes. All these centres used ${ }^{125} \mathrm{I}$ seeds (i.e. no ${ }^{103} \mathrm{Pd}$ or ${ }^{131} \mathrm{C}$ s seeds). Table 3 shows the selection criteria used in 2007 and the resulting distribution of patient numbers by risk and T-classification. Two institutions reported the occasional use of ${ }^{125} \mathrm{I}$ treatment in combination with EBRT. Reported dose levels for monotherapy treatment were 144 Gy or 145 Gy, except for 1 institution (160 Gy). Two institutions reported the use of both stranded and loose seeds, while 10 used stranded

Table 1. Total number of patients treated in 2007 and 2002, and the number of institutions performing the technique

\begin{tabular}{|c|c|c|c|c|c|c|}
\hline \multirow{2}{*}{$\begin{array}{l}\text { Sites and number } \\
\text { of treatments }\end{array}$} & \multicolumn{4}{|c|}{2007} & \multirow{2}{*}{$\frac{2002}{\text { No. of patients }}$} & \multirow{2}{*}{$\frac{2007}{\text { Cancer incidence }}$} \\
\hline & No. of patients & $\%$ of incidence & Institutions & Average/Institution & & \\
\hline Lung - bronchus & 3 & $0.03 \%$ & 2 & 1.5 & 30 & 10533 \\
\hline Gyn. - endometrium & 211 & $11.8 \%$ & 13 & 16.2 & 266 & 1783 \\
\hline Gyn. - cervix & 305 & $41.6 \%$ & 16 & 19.1 & 304 & 734 \\
\hline Gyn. - vagina/vulva & 80 & $21.3 \%$ & 14 & 5.7 & 16 & 376 \\
\hline Head and neck & 83 & $9.3 \%$ & 7 & 11.9 & 79 & 890 \\
\hline Breast & 79 & $0.6 \%$ & 4 & 19.8 & 72 & 12930 \\
\hline Anal-canal & 26 & $18.8 \%$ & 7 & 3.7 & 33 & 138 \\
\hline Prostate $-1^{125}$ & 1030 & $10.7 \%$ & 13 & 79.2 & 419 & 9588 \\
\hline Prostate - |r192 & 20 & & 1 & 20.0 & 39 & \\
\hline Prostate - HDR & 22 & & 1 & 22.0 & 0 & \\
\hline Oesophagus & 333 & $20.2 \%$ & 18 & 18.5 & 300 & 1651 \\
\hline Bladder & 49 & $1.8 \%$ & 9 & 5.4 & 56 & 2688 \\
\hline Skin-tumour & 14 & & 3 & 4.7 & 44 & \\
\hline Skin - keloid & 26 & & 5 & 5.2 & & \\
\hline Eye & 143 & $76.5 \%$ & 6 & 23.8 & 153 & 187 \\
\hline Pediatric & 3 & & 1 & 3.0 & & \\
\hline Other & 33 & & 6 & 5.5 & 173 & \\
\hline Total & 2460 & & & & 1984 & \\
\hline
\end{tabular}


Table 2. Breakdown of the 596 cases of gynaecological patients treated in 2007

\begin{tabular}{|c|c|c|c|c|}
\hline Treatment intent & Preoperative & Postoperative & Definitive & Total \\
\hline Endometrium & 0 & 208 & 3 & 211 \\
\hline Cervix & 11 & 25 & 269 & 305 \\
\hline Vagina & 9 & 8 & 59 & 76 \\
\hline Vulva & 0 & 0 & 4 & 4 \\
\hline Total & 20 & 241 & 335 & 596 \\
\hline in \% & $3.4 \%$ & $40.4 \%$ & $56.2 \%$ & $100.0 \%$ \\
\hline
\end{tabular}

seeds only. Treatment planning was done prior to treatment ( 2 centres); intraoperatively (6 centres); intraoperative preplanning (9 centres); or interactively (11 centres). We should note that more than one answer was permitted as an institution may decide to use more than one technique. Only one institution reported using low-fractionated HDR afterloading treatments for prostate cancer, either as a monotherapy or in a combination with EBRT.

\section{Discussion and Conclusions}

This report summarizes the results of the participation of one country in the GEC-ESTRO Patterns of Care Study for Brachytherapy in 2002 and 2007. The general results of the 2002 PCBE data collection on BT infrastructure and practices in the European area were published by Guedea et al. in 2007 [3] and 2008 [15]. In another paper, the general 2007 results of the project will be addressed [9]. Compared to 2002, the 2007 survey was only slightly changed. The questions and definitions used in the new version were defined more precisely to avoid ambiguity in interpretation. Furthermore, a number of questions were added, including detailed questions on lung, breast, gynaecology, and prostate treatments. A $100 \%$ response rate was achieved from the 21 centres in The Netherlands.

\section{Patients, applications, and staff}

In general, the PCB data from the Dutch institutions showed a large variation, both between institutions and in some items viewed over the 5-year period. The changes that can be observed on a national scale are more gradual. The cancer incidence registry of the comprehensive cancer centres in The Netherlands shows an overall average annual increase of invasive tumours of $3.4 \%$ calculated over a 5 -year period, from 73675 patients in 2002 to 86162 patients in 2007 [10]. The number of radiation therapy patients recorded in the present study closely follows this growth: from 41528 in 2002 to 47594 in 2007, an annual increase of approximately $3 \%$. A total of 2460 new BT patients was recorded in 2007, an annual increase of $4.8 \%$ compared to the figure of 1984 patients in 2002, which was largely due to the increase in prostate procedures. These average data correlate with the expected growth rate due to a combination of population increase and aging: often an annual growth rate of $2.5-3 \%$ is used in decision making projections to the future. However, a few of the - mainly smaller - institutions demonstrated a decrease in total RT procedures, while others showed large increases (up to $+40 \%$ ).
Table 3. Selection criteria, number of patients by TNM staging system for all 2007 prostate patients. Note that the total number of patients deviates slightly from the numbers elsewhere in this overview due to the fact that not all institutions gave a complete list

\begin{tabular}{lcc}
\multicolumn{2}{l}{ Risk classification prostate patients } & Total \\
\hline Low risk (T1/T2) & $54.2 \%$ & 563 \\
\hline Intermediate risk (T1/T2) & $32.9 \%$ & 341 \\
\hline High risk (T1/T2) & $10.9 \%$ & 113 \\
\hline T3 & $2.0 \%$ & 21 \\
\hline Total & $100 \%$ & 1038
\end{tabular}

The overall percent increase in average staffing level for radiation oncologists $(29 \%)$ was about twice the overall average growth number of RT procedures $(15 \%)$, meaning that the patients per radiation oncologist ratio has decreased in these 5 years. The number of radiation oncologist performing BT in The Netherlands remained practically unchanged.

\section{Resources}

In general, institutions have much more capacity than strictly needed: thirteen out of 21 institutions were able to perform the ${ }^{125}$ I seed implantation technique with up to a total of 1030 patient cases in 2007. If we simply divide the remaining number of BT patients in 2007 (2460 - $1030=$ $=1430$ ) by the total number of available afterloaders (26; 15 HDR and 11 PDR), then the actual workload per afterloader is close to 1 case per week. The capacity to treat patients with an HDR afterloader is in principle much higher: it is possible to use an HDR afterloader for 2 cases per day or even many more per day in a busy, dedicated, and efficiently organised BT unit.

The replacement of the former generation of LDR afterloading equipment with modern HDR and PDR afterloaders is striking. One clear reason for this is the announced discontinuation of servicing of a widely-used ${ }^{137} \mathrm{Cs}$ afterloading device. A similar observation was recently made by Pearce et al. [16] who studied the current practice of the treatment of carcinoma of the cervix in Canadian centres. They specifically were interested in how the phasing-out of low dose rate technology from the commercial BT market was changing practice. In addition we observed that other types of micro-source afterloaders 
operating with either LDR ${ }^{137} \mathrm{Cs}$ seed strings or ${ }^{192} \mathrm{Ir}$ wires/seed strings have nearly disappeared from Dutch institutions. A simultaneous increase in PDR devices and, to some extent, HDR afterloaders was observed, a development that is also expected to happen in other countries. Any further changes in the number and types of afterloaders in the near future is likely to be related to the replacement of the existing equipment due to aging only, as for most institutions the currently available HDR and PDR equipment provide more than sufficient capacity to meet their needs. The Dutch strategy to replace LDR techniques with PDR techniques seems logical from the point of view of keeping 'equivalency' in dose rate, but is typically a regional phenomenon in The Netherlands although it also occurs in other countries, such as France. Quite often institutions - e.g. in developing countries but evenly well in other developed parts of the world - choose instead to work only with HDR equipment, mainly for economic reasons.

The data on available imaging modalities reported here support the observation that changes towards the high standard imaging technologies in the BT field penetrate only relatively slowly into clinical practice. The introduction of CT and MR based treatment planning in BT is occurring at a relatively slow pace in this country. The use of CT in BT treatment planning increased from 6 to 10 institutions, MRI scanning from 3 to 4 centres, and one institution reported using PET scanning in 2007. The use of ultrasound increased in parallel with the growth of prostate seed implant technique. Use of 3D imaging is expected to grow in parallel with the implementation of modern recommendations for dosimetry and with participation in international trials, e.g. in gynaecology (the Embrace study), and in prostate treatments $[13,14,17]$. In addition to the data on resources provided in this section, it should be noted that 6 (out of 16) institutions reported following the GEC-ESTRO BT recommendations for treatment of carcinoma of the cervix. This number is expected to increase even further in the next few years and should become apparent in a next survey [18].

A large increase was observed in the number of TPSs used for BT. In most cases a dedicated TPS is introduced in addition to a conventional system when the prostate treatment technique is introduced. Furthermore, a small number of institutions have shown interest in using in vivo dosimetry in BT, with an increase from 2 to 4 institutions over the 5-year interval. In the Dutch 2007 data, there is no information available on the introduction or use of other radionuclides such as ${ }^{60} \mathrm{Co},{ }^{170} \mathrm{Tm}$ or ${ }^{169} \mathrm{Yb}$. Use of such radionuclides may appear in new surveys in the next decade. There seems to be no future for endovascular treatments, as the dedicated equipment has completely disappeared from the BT field in this country, in line with the paradigm shift reported by Van Limbergen and Trepuranemi [19].

\section{Site specific patient numbers}

With regards to the numbers of patients treated with BT, the general picture is one of stability in The Netherlands. The number of BT patients compared to the total number of new RT patients is close to $5 \%$. For most tumour locations - gynaecological tumours, head and neck, bladder, breast, oesophagus, and eye tumours - changes between 2002 and 2007 were small and generally followed growth in incidence. However, it should be noted that, except for eye tumours (77\%), cervix (42\%), oesophageal cancer $(20 \%)$, and anal canal (19\%), the number of BT procedures per institution related to the respective cancer incidence is very small (Table 1). Endovascular treatments disappeared from the spectrum as the coated stents were shown to be superior [19].

One clear exception, however, is the huge increase in the number of ${ }^{125} \mathrm{I}$ seed implant cases for prostate treatment, a practice that grew since 2002 by $146 \%$ to a total of 1030 cases in 13 Dutch institutions over this 5 year period. The 2002 questionnaire was apparently carried out in a period in which the number of institutions performing this technique developed a rapid expansion. One explanation for this is found in the reimbursement system for this type of treatment in the Dutch health care system, which was changed at that time and since then allowed for full reimbursement for the costly radioactive materials. Another essential reason is likely the growing clinical evidence for image-guided prostate BT [20]. The rapid growth observed in the Dutch centres fits perfectly well in the study on the use of the prostate implant technique in Europe performed by the GEC-ESTRO groups Probate and Braphyqs, described previously by Hoskin and Venselaar in 2007 [21]. Based on the number of diagnosed prostate cancer patients of 9588 cases in 2007 [10], we calculate that in $200710.7 \%$ of all prostate patients were treated with this modality. Some further growth in prostate BT treatments is possible if the other hospitals/institutions decide to startup a prostate programme. Introduction of other techniques as high-intensity focused ultrasound (HIFU) and robotic surgery, however, may counteract this possible growth.

\section{Detailed site specific questions}

Although the questionnaire contained specific questions on a number of different treatment sites, in many cases the responses cannot be properly analysed. For example, the number of treated lung cancer patients in The Netherlands was very low, deviating from what is reported from surveys in some other countries [22-24]. Furthermore the majority of cases were reported from a single centre.

The number of institutions performing breast BT was similarly low: with $85 \%$ of the 79 breast cases coming from one institution (out of 4 institutions using the technique) no general conclusions can be drawn. From the survey results it is not at all clear to define what is the influence of the upcoming evidence of accelerated partial-breast irradiation (APBI) for the number of patients that is going to be treated in the near future in this country with interstitial or intracavitary balloon techniques. A recent overview of patient selection for APBI after breast-conserving surgery was presented as recommendations of the GEC-ESTRO breast cancer working group, based on clinical evidence [25]. Further clinical research is promoted by this group focusing on controversial issues in the treatment of early-stage breast carcinoma. But the future of breast BT 
in The Netherlands is difficult to predict due to possible other conformal techniques with EBRT.

It is expected that a similar effect of the clinical evidence for improved outcome of image-guided implant techniques as indicated above for prostate treatments will favour the future use of BT for gynaecology [26]. Major changes may result with the increased use of MR and CT based 3D/4D treatment planning, in particular for definitive techniques. In regard to treatment intent, the number of radical treatments can be expected to remain stable, while it remains unclear if the results of the PORTEC-2 with BT compared to EBRT will change the patterns of practice in postoperative endometrial treatment [11].

With regard to prostate implants, the use of the seed technique seems to be generally uniform throughout the country. In addition, there is only one centre with PDR, and one with HDR as treatment modality. No other radionuclides were used for seeds than ${ }^{125}$ I. Dose prescription was uniform at the 144-145 Gy level, with only one exception. In general, modern intraoperative or interactive treatment planning in the OR was used. As a shortcoming in the survey with regard to prostate implant dosimetry it is noted that treatment margin or other details about dose or volume specification was not included in the questions.

\section{Research and education}

Eight institutions in this survey have reported participating in a total of 4 prospective clinical trials and 6 local study protocols. We suspect that these answers underreports important clinical and physics research work being performed in The Netherlands in the field of BT. Recent studies for example showed clear interest from different groups in a wide variety of topics such as radiobiological dose adding [27]; 3D image guided optimisation for the cervix ring applicator [28]; treatment of bladder cancer using BT [29,30]; and head and neck implant techniques [31].

Four of the 20 institutions reported having special BT education programmes that ranged from practical training of residents, sending trainees to teaching courses (e.g., ESTRO), and organisation of dedicated PDR workshops. The answers were scarce, quite variable, and pointing in different directions. All post-academic $(8 \times$ in The Netherlands) education programmes have clear programs for their residents with regard to BT training, but this is not reflected in the answers to the survey. No reference was made to any of the ESTRO documents in which training is discussed $[1,32,33]$.

\section{General comments}

The average number of BT patients per institution is approximately 100 patients per year in The Netherlands. Table 1 makes clear that, for several treatment sites, BT is applied with relatively low numbers of patients. The reported patient numbers for head and neck, anal canal, paediatrics, bladder and eye interventions ranged from 3-20 patients per year per institution, with few exceptions. However, these techniques absolutely require experienced teams working closely with the surgeons (except for anal canal). Treatment planning for these techniques is complex and the use of optimal imaging is recommended. These sites are therefore suited for concentration in one or a few specialised centres in a country and thus for referrals from other, peripheral institutions. As a first step in defining quality requirements, the Dutch society for radiation oncology recommended that centres perform a minimum of 35 prostate implants per year to maintain proper quality. The smallest number of patients treated for prostate cancer at any one institution in 2007 was 27 . Similar recommendations might be defined for the complex treatment sites cited above, taking into account complexity of technique, site-specific cancer incidence, available expertise, regional function of the centre, etcetera.

The survey helps to identify discrepancies between recommendations and practice. The fact that this type of survey is made on an international scale in combination with a detailed analysis of the local situation allows the national society to develop guidelines for high quality work in specific areas. Establishing expertise based on a minimum number of treatments per institution/year for certain sites may be one of those guidelines or quality indicators. Survey questions for specific treatment sites (e.g. on the practise of dose prescription and margins, the use of specific imaging modalities) may similarly be helpful in determining the uniformity of BT procedures.

These survey results, combined with data on cancer incidence and related developments and when compared with data from other countries or regions, allow us to identify general trends and possible regional differences in practices. The results may then support the continuation of the PCB study to document further developments and changes in the field as well as any notable increases or decreases in patient numbers per tumour category. Continuation of this study is recommended to improve RT provision on rational grounds throughout the European area and beyond and as a model for health care planning.

\section{Acknowledgements}

The authors wish to thank the leaders of the radiation therapy facilities in The Netherlands for their cooperation in completing the questionnaires. In particular, we thank the contact persons who did a great job in collecting the data, especially considering the difficulties of data collection, much of which must be retrieved retrospectively. The valuable comments to improve the manuscript by Prof. R. Pötter were highly appreciated. The PCB project was supported financially by Oncura, Nucletron, and Varian, as well as by a grant from the Spanish Ministry of Health (FIS PI070889).

\section{References}

1. Bentzen SM, Heeren G, Cottier B et al. Towards evidence-based guidelines for radiotherapy infrastructure and staffing needs in Europe: the ESTRO QUARTS project. Radiother Oncol 2005; 75: 355-365.

2. Slotman BJ, Cottier B, Bentzen SM et al. Overview of national guidelines for infrastructure and staffing of radiotherapy. ESTRO-QUARTS: Work package 1. Radiother Oncol 2005; 75: 349-349. 
3. Guedea F, Ellison T, Venselaar J et al. Overview of brachytherapy resources in Europe: A survey of patterns of care study for brachytherapy in Europe. Radiother Oncol 2007; 82: 50-54.

4. Venselaar J, Guedea F, Slotman BJ et al. Results of the PCBE questionnaire-2002 in The Netherlands. Radiother Oncol 2004; 71, Supp 2: S2-S3 (Abstract).

5. Peiffert D, Mazeron JJ, Guedea F et al. Brachytherapy in France in 2002: results of the ESTRO PCBE questionnaire. Cancer/Radiothérapie 2007; 11: 146-149 (Abstract).

6. Torrecilla JL, Guedea F, Heeren G et al. Patterns of care for brachytherapy in Europe results in Spain. Clin Translat Oncol 2006; 8: 362-368.

7. Guedea F, Ventura M, Polo A et al. Patterns of care for brachytherapy in Europe (PCBE) in Spain and Poland: Comparative results. Rep Pract Oncol Radiother 2007; 12: 39-45.

8. Radiologists. BoFoCORCo. The Role and Development of Brachytherapy Services in the United Kingdom. Accessed at: http://www.rcr.ac.uk/docs/oncology/pdf/brachytherapy.pdf, London: The Royal College of Radiologists 2001.

9. Guedea F, Venselaar J, Hoskin P et al. Patterns of Care for Brachytherapy in Europe: Updated Result. Radiother Oncol. (Accepted, ahead of publication, last accessed Nov 22 2010).

10. http://www.ikcnet.nl/uploaded/docs/Landelijk/cijfers/ incidentie\%202007/A01_NL.xls, Comprehensive Cancer Centres in The Netherlands, Netherlands Cancer Registry 1989-2007. Last accessed November 22, 2010.

11. Creutzberg CL, van Putten WL, Koper PC et al. Surgery and postoperative radiotherapy versus surgery alone for patients with stage-1 endometrial carcinoma: Multicentre randomised trial. PORTEC Study Group. Postoperative Radiation Therapy in Endometrial Carcinoma. Lancet 2000; 355: 1404-1411.

12. Creutzberg CL, van Putten WLJ, Koper PC et al.; for the PORTEC Study Group. The morbidity of treatment for patients with stage I endometrial cancer: results from a randomized trial. Int J Radiat Oncol Biol Phys 2001; 51: 1246-1255.

13. Pötter R, Haie-Meder C, Van Limbergen E et al. Recommendations from gynaecological (GYN) GEC ESTRO working group (II): Concepts and terms in 3D image-based treatment planning in cervix cancer brachytherapy - 3D dose volume parameters and aspects of 3D image-based anatomy, radiation physics, radiobiology. Radiother Oncol 2006; 78: 67-77.

14. Haie-Meder C, Pötter R, Van Limbergen E et al. Recommendations from Gynaecological (GYN) GEC-ESTRO Working Group (I): concepts and terms in 3D image based 3D treatment planning in cervix cancer brachytherapy with emphasis on MRI assessment of GTV and CTV. Radiother Oncol 2005; 74: 235-245.

15. Guedea F, Ventura M, Mazeron JJ et al. Patterns of care for brachytherapy in Europe: facilities and resources in brachytherapy in the European area. Brachytherapy 2008; 7: 223-230.

16. Pearce A, Craighead P, Kay I, Traptow L et al. Brachytherapy for carcinoma of the cervix: A Canadian survey of practice patterns in a changing era. Radiother Oncol 2009; 91: 194-196.

17. Salembier C, Lavagnini P, Nickers P et al.; on behalf of the PROBATE group of GEC ESTRO. Tumour and target volumes in permanent prostate brachytherapy: A supplement to the ESTRO/EAU/EORTC recommendations on prostate brachytherapy. Radiother Oncol 2007; 83: 3-10.

18. Erickson B, Eifel P, Moughan J et al. Patterns of brachytherapy practice for patients with carcinoma of the cervix (19961999): a patterns of care study. Int J Radiat Oncol Biol Phys 2005; 63: 1083-1092.

19. Van Limbergen E, Trepuranemi P. Is this the swan song of endovascular brachytherapy? Radiother Oncol 2007; 82: 1-4.

20. Polo A, Salembier C, Venselaar J et al. on behalf of the PROBATE group of the GEC ESTRO. Review of intraoperative imaging and planning techniques in permanent seed prostate brachytherapy. Radiother Oncol 2010; 94: 12-23.

21. Hoskin PJ, Venselaar J. Prostate brachytherapy in Europe: growth, practice and guidelines. Radiother Oncol 2007; 83: 1-2.

22. Nag S, Kelly JF, Horton JL et al. Brachytherapy for carcinoma of the lung. Oncology 2001; 15: 371-381.

23. Tai P, Yu E, Battista J et al. Radiation treatment of lung cancer - patterns of practice in Canada. Radiother Oncol 2004; 71: 167-174.

24. Demiral AN, Alicikus ZA, Işil Ugur V et al. Patterns of care for lung cancer in radiation oncology departments of Turkey. Int J Radiat Oncol Biol Phys 2008; 72: 1530-1537.

25. Polgár C, Van Limbergen E, Pötter R et al.; on behalf of the GEC-ESTRO Breast Cancer Working Group. Patient selection for accelerated partial-breast irradiation (APBI) after breastconserving surgery: Recommendations of the Groupe Européen de Curiethérapie-European Society for Therapeutic Radiology and Oncology (GEC-ESTRO) breast cancer working group based on clinical evidence. Radiother Oncol 2010; 94: 264-273.

26. Pötter R, Dimopoulos J, Georg P et al. Clinical impact of MRI assisted dose volume adaptation and dose escalation in brachytherapy of locally advanced cervix cancer. Radiother Oncol 2007; 83: 148-155.

27. Van de Kamer JB, De Leeuw AAC, Moerland MA et al. Determining DVH parameters for combined external beam and brachytherapy treatment: 3D biological dose adding for patients with cervical cancer. Radiother Oncol 2010; 94: 248-253.

28. Jürgenliemk-Schulz I-M, Lang S, Tanderup K et al.; on behalf of the Gyn GEC ESTRO network. Variation of treatment planning parameters (D90 HR-CTV, D2cc for OAR) for cervical cancer tandem ring brachytherapy in a multicentre setting: Comparison of standard planning and 3D image guided optimisation based on a joint protocol for dose-volume constraints. Radiother Oncol 2010; 94: 339-345.

29. Van der Steen-Banasik E, Ploeg M, Witjes JA et al. Brachytherapy versus cystectomy in solitary bladder cancer: a case control, multicentre, East-Netherlands study. Radiother Oncol 2009; 93: 352-357.

30. Pos FJ, Van Tienhoven G, Hulshof MC et al. Concomitant boost radiotherapy for muscle invasive bladder cancer. Radiother Oncol 2003; 68: 75-80.

31. Kolkman-Deurloo I-KK, De Kruijf WJM, Levendag PC. On-line implant reconstruction in HDR brachytherapy. Radiother Oncol 2006; 78: 53-59.

32. Pötter R, Van Limbergen E, Dries W et al. Recommendations of the EVA GEC ESTRO Working Group: prescribing, recording, and reporting in endovascular brachytherapy. Quality assurance, equipment, personnel and education. Radiother Oncol 2001; 59: 339-360.

33. Röttinger E, Barrett A, Leer JW, for the European Board of Radiotherapy. Guidelines for the infrastructure of training institutes and teaching departments for radiotherapy in Europe. Accessed at: http:/ / www.estro-education.org/europeantraining/Documents/GITITDRE.pdf. 